• 研究报告・

\title{
青藏高原高寒草甸植物花寿命对传粉环境的响应
}

\author{
王玉贤 ${ }^{1}$ 刘左军 $^{1^{*}}$ 赵志刚 ${ }^{2}$ 侯 盟 $^{2}$ 张小瑞 $^{1}$ 吕婉灵 ${ }^{1}$ \\ 1 (兰州理工大学生命科学与工程学院, 兰州 730050) \\ 2 (兰州大学生命科学学院, 草地农业生态系统国家重点实验室, 兰州 730000)
}

摘要: 花寿命是指花保持开放且具有功能的时间长度。不同物种的花寿命具有显著的差异, 但一定程度上的可塑 性反映了植物对传粉环境变化的适应性。本研究以青藏高原高寒草甸不同海拔 $(2,900 \mathrm{~m}$ 和 $3,600 \mathrm{~m}$ )的11种开花植物 为研究对象, 通过套袋、补充授粉以及自然授粉处理分别测量了植物的潜在花寿命、最短花寿命以及实际花寿命。 分析了不同海拔植物花寿命的变异, 以及对套袋处理(潜在花寿命与实际花寿命之差)和补充授粉处理(实际花寿命 与最短花寿命之差)的响应及其可塑性(潜在花寿命与最短花寿命之差)的变异。结果表明: 无论是高海拔还是低海 拔, 套袋显著延长了花寿命, 而补充授粉显著缩短了花寿命, 即, 潜在花寿命>实际花寿命>最短花寿命。高海拔群 落中植物的实际花寿命和潜在花寿命均显著延长, 而最短花寿命在海拔间差异不显著。相对于低海拔群落, 高海 拔植物的花寿命对补充授粉处理更敏感, 花寿命的可塑性更大。本研究说明传粉者稀少的高寒环境中, 植物通过 提高花寿命及其可塑性来增加授粉机会, 获得更高的适合度。

关键词: 高寒草甸; 花寿命; 可塑性; 授粉; 海拔

\section{Responses of floral longevity to pollination environments in 11 species from two alpine meadows}

\author{
Yuxian Wang ${ }^{1}$, Zuojun $\mathrm{Liu}^{1 *}$, Zhigang Zhao ${ }^{2}$, Meng $\mathrm{Hou}^{2}$, Xiaorui Zhang ${ }^{1}$, Wanling $\mathrm{Lü}^{1}$ \\ 1 School of Life Science and Engineering, Lanzhou University of Technology, Lanzhou 730050 \\ 2 School of Life Sciences, Lanzhou University, State Key Laboratory of Grassland Agro-Ecosystems, Lanzhou 730000
}

\begin{abstract}
Floral longevity, the length of time that a flower remains open and functional, varies greatly among species. The high plasticity of floral longevity can reflect an adaptation to variable pollination environments. In the alpine meadows of Qinghai-Tibetan Plateau (2,900 m vs. 3,600 m), we assessed how potential floral longevity, the shortest floral longevity and the actual floral longevity of 11 species varied under treatments of different pollination contexts. We modeled the response of floral longevity to pollinator exclusion (potential floral longevity minus actual floral longevity) and supplemental pollination (actual longevity minus shortest longevity), and the plasticity of floral longevity (potential longevity minus shortest longevity) at high and low altitude habitats. We found that the plants at the high-altitude community had longer potential floral longevity compared to the low-altitude community, while the shortest floral longevity was not significantly different. Furthermore, pollinator exclusion significantly increased flower longevity, while supplemental pollination significantly decreased floral longevity (i.e. the potential floral longevity $>$ the actual floral longevity $>$ the shortest floral longevity) in both high and low-altitude habitats. In comparison with the low-altitude community, high-altitude plants exhibited higher plasticity of floral longevity. Overall, our results suggest that greater plasticity of floral longevity may increase the opportunity for pollination and thus aid fitness at higher altitudes where pollinators are scarce and unpredictable.
\end{abstract}

Key words: alpine meadow; floral longevity; plasticity; pollination; altitude 
异(Primack, 1985; Ashman \& Schoen, 1994)。植物的 花寿命由雌雄适合度的自然增长率(花粉的落置与 移出的速率)与维持花开放的资源消耗之间的平衡 决定(Ashman \& Schoen, 1994; Schoen \& Ashman, 1995)。单花寿命影响植物同时开放的总花数目, 进 而影响花展示的大小及其对传粉者的吸引, 最终会 影响植物的交配类型、传粉者访问的次数、花粉接 收与扩散的数量和质量等(Primack, 1985; Harder \& Johnson, 2005), 这些因素都与植物的繁殖和适合度 紧密相关。此外, 一些研究还发现花寿命与种子和 果实的产出之间存在平衡 (Ashman \& Schoen, 1997)。因此，花寿命作为一种繁殖特征影响着植物 能否繁殖成功, 对繁殖策略的进化有着重要的意义 (张志强和李庆军, 2009)。

不同物种的花寿命是经过长期进化形成的, 它 们能够稳定地遗传给后代, 是一种适应性的、可遗 传的花特征(Primack, 1985)。不同物种可能展现出 遗传决定的最短花寿命和最长花寿命 (Evanhoe \& Galloway, 2002; Gerlinde \& Johanna, 2010)。最短花 寿命是指通过人工补充授粉使得花的雌性功能完 全实现时, 所呈现的最短雄性功能期(Evanhoe \& Galloway, 2002); 而最长花寿命则是指通过套袋等 措施隔离传粉者, 使花朵在未授粉状态下保持开放 的时间长度(Rathcke, 2003), 也称之为潜在花寿命 (Gerlinde \& Johanna, 2010)。将花朵在自然授粉情况 下开放的时间长度认为是实际花寿命(Arroyo et al, 2013)。

尽管花寿命是一种具有适应性的、可遗传的特 征, 它们在一定程度上仍具有可塑性(Clark \& Husband, 2007)。表型可塑性是植物适应不同生境的重 要生态对策(Sultan, 2000)。通过表型可塑性, 植物 可以最大限度地获取资源并进行再分配，从而实现 资源的有效利用，提高植物适合度。而花寿命的可 塑性主要表现在它随着传粉环境的波动而延长或 缩短, 这也在很多研究中得到了证实(Evanhoe \& Galloway, 2002; Spigler, 2017)。花寿命的可塑性通 常由两个部分组成, 即花寿命对隔离传粉者处理的 响应和对补充授粉处理的响应。花寿命对隔离传粉 者的响应通常是指在套袋隔离传粉者的条件下花 寿命所能延长的程度, 即潜在花寿命与实际花寿命 之差。花寿命对补充授粉处理的响应则指在经过人 工补充授粉后花寿命所能缩短的程度, 即实际花寿
命与最短花寿命之差。而这两者之和即为花寿命的 可塑性，即潜在花寿命与最短花寿命之差，也就是 指花寿命的最大变化范围(Duan et al, 2007; Trunschke \& Stöcklin, 2016)。

植物单花寿命的长短不仅受到物种自身因素 (如生长习性、植物特性、花大小等; Primack, 1985; Gao et al, 2015)的影响, 还与其所生长环境的生物 (传粉者种类、丰富度等)与非生物(温度、降水量等) 因素(Primack, 1985; Ashman \& Schoen, 1994)有关。 越来越多的授粉实验表明，植物单花寿命与该花的 授粉强度呈显著的负相关(Evanhoe \& Galloway, 2002; Castro et al, 2008; Spigler, 2017), 即随着柱头 上落置的花粉数量的增加，植物花寿命会缩短 (Rathcke, 2003; Giblin, 2005; Itagaki \& Sakai, 2006); 而在套袋隔离传粉者时，花寿命将会延长(Vesprini \& Pacini, 2005; Spigler, 2017)。这是因为在授粉的情 况下雌性适合度在短期内就获得成功并诱导了花 的衰老(Aximoff \& Freitas, 2010; Weber \& Goodwillie, 2013)。而在自然群落中, 对于异花传粉的植物 来说, 传粉通常是通过传粉者访花实现的。一些野 外实验显示, 植物的花寿命受到传粉者种类的影响 (Primack, 1985; Teixido \& Valladares, 2015)。这可能 是由于植物花朵与不同传粉者之间的匹配度不同 导致了不同的传粉效率，进而影响了雌性适合度获 得的时间长度, 并最终影响了植物的花寿命。同时, 当传粉者丰富度较低时, 植物通常会延长花寿命 (Gerlinde \& Johanna, 2010; Marques \& Draper, 2012), 为传粉者提供更多的访花机会, 从而使依赖动物传 粉的植物获得繁殖成功, 这是一种繁殖保障的机制 (Rathcke, 2003; Castro et al, 2008)。另外, 随着温度 的降低, 植物的花寿命呈现延长趋势(Clark \& Husband, 2007; Arroyo et al, 2013)。这可能是由于在低 温条件下，传粉者的丰富度及活力等明显下降，导 致花朵的授粉强度下降的缘故 (Primack, 1985; Rathcke, 2003); 但也有可能是低温导致植物花朵发 育缓慢引起的(Arroyo et al, 1981; Rathcke, 2003; Vega \& Marques, 2015)。

总的来说, 植物的花寿命与传粉者的可利用强 度有关，即花寿命随着传粉者的可利用强度的下降 而延长(Primack, 1985; Trunschke \& Stöcklin, 2016)。

在不同的植物生境中, 传粉者的种类、丰富度 及活力均存在很大差异(Bingham \& Orthner, 1998; 
Blionis \& Vokou, 2001)。通常, 相对于低海拔生境, 在高海拔生境中, 温度较低, 传粉者的种类、丰富 度以及活力都比较低(Arroyo et al, 1981; Stratton, 1989; Bingham \& Orthner, 1998)。因此, 随着海拔梯 度的升高, 花寿命普遍延长(Teixido \& Valladares, 2015; Trunschke \& Stöcklin, 2016)。一个物种花寿命 的可塑性能够反映其对不稳定的随机传粉环境的 适应能力, 而大多数对花寿命的研究主要集中于影 响花寿命的因素, 只有少数研究关注植物花寿命的 可塑性大小。

本研究通过传粉处理探讨了青藏高原东部不 同海拔高寒草甸群落的主要开花植物花寿命的长 短及其可塑性变异, 主要回答下列问题: (1)相对于 低海拔植物群落, 高海拔群落中植物花寿命是否普 遍延长？(2)高海拔群落植物花寿命的可塑性是否 高于低海拔的? 并进一步预测, 低海拔群落中植物 的花寿命对套袋处理的响应更快, 而高海拔群落中 植物花寿命对补充授粉处理的响应更快。

\section{材料与方法}

\section{1 研究样地与物种}

本研究于2017年6-7月在兰州大学高寒草甸与 湿地生态系统定位站的合作站 $\left(102^{\circ} \mathrm{N}, 34^{\circ} 70^{\prime} \mathrm{E}\right.$, 低海拔样地, 约 $2,900 \mathrm{~m})$ 与阿孜站 $\left(35^{\circ} 58^{\prime} \mathrm{N}\right.$, $101^{\circ} 53^{\prime} \mathrm{E}$, 高海拔样地, 约3,600 m)进行, 在两个站 的高寒草甸样地分别选取一个 $50 \mathrm{~m} \times 50 \mathrm{~m}$ 的样区。

选取两个样区中的主要开花物种作为研究对 象。主要为瑞香科的狼毒(Stellera chamaejasme), 豆 科的高山豆(Tibetia himalaica), 蓄薇科的莓叶委陵 菜 (Potentilla fragarioides)、鹅线委陵菜 (P. anserina)、华西委陵菜(P. potaninii)、野草莓(Fragaria vesca), 毛茛科的小花草玉梅(Anemone rivularis var. flore-minore)、针裂银莲花(A. obtusiloba) 和高原毛茛 (Ranunculus tanguticus), 菊科的蒲公英(Taraxacum mongolicum) 以及陇牛儿苗科的甘青老鹳草 (Geranium pylzowianum)。其中, 莓叶委陵菜、针裂 银莲花、高原毛茛、蒲公英以及甘青老鹳草这 5 种 是高海拔和低海拔样区共有物种, 它们都为多年生 植物, 主要由比较泛化的传粉者传粉。其中针裂银 莲花是以自交为主的混合交配类型(胡春等, 2013); 高原毛莨和小花草玉梅是兼性自交交配系统(赵志 刚和杜国祯，2003); 甘青老鹳草为异交物种; 蒲公
英为有性生殖和无融合生殖混合繁殖系统(张建, 2013)。6月22日至7月11日标记、处理及观察低海拔 样区10个物种。7月12日至7月25日标记、处理及观 察高海拔样区6个物种，所有实验观测都在每个物 种的盛花期进行。

\section{2 研究方法}

每个研究种群随机选取 10 组植株, 每组包含 3 株含有花苞的植株, 其中蒲公英和狼毒的研究种群 每组包含 2 株含有花苞的植株, 因为这两种植物所测 的是花序寿命，不对其进行补充授粉处理。为了减 少花朵位置对花寿命的效应，对处于每个植株相同 位置的1朵花苍进行标记(每个植株标记1朵花，每 个种群每个处理有 10 朵花, 总共标记了 460 朵花), 然后对每组标记的花苞分别进行如下处理: (1)套袋 处理，其目的是为了测得在隔离传粉者后的花寿命， 即潜在花寿命; (2)补充授粉处理, 其目的是为了测 得在最大授粉强度下的花寿命，即最短花寿命; (3) 自然授粉，其目的是为了测得花朵在自然授粉状态 下的花寿命, 即实际花寿命。为了避免传粉者访花, 所有套袋处理的花苍，都用无纺布袋在花还未开放 时进行套袋。所有进行补充授粉处理的花朵都用样 区外收集的相同物种的成熟花粉，在花朵一进入雌 性期就进行补充授粉。其中钝裂银莲花和高原毛茛 连续授粉 3 天, 莓叶委陵菜、鹅线委陵菜、野草莓、 华西委陵菜和小花草玉梅连续授粉 2 天，而甘青老 鹳草和高山豆只需补充授粉 1 天。

每天早上9:00和下午17:00观察每个实验花朵 的开花状况，记录每一朵花的开花时间及闭花时 间。开花时间指花苞开始张开的时间，而闭花时间 则指花瓣已开始调落, 或用手指轻轻一碰花瓣就开 始掉落的时间。

\section{3 数据处理}

在计算花寿命的时候，如果开花时间在12:30 之前记为 1.0 天, 12 : 30 之后记为 0.5 天; 花调谢时间 在12:30之前记为 0.5 天, $12: 30$ 之后记为 1.0 天。

首先，运用线性混合模型的最大似然法(LME) 分析不同海拔高度及授粉处理对花寿命的影响，其 中花寿命作为因变量, 物种、授粉处理、海拔及它 们的交互项作为固定效应，植物的分组则作为随机 效应。

其次, 为了比较同一海拔高度下不同授粉处理 对花寿命的影响, 在群落水平上, 运用一般线性模 
型检验授粉处理对花寿命的影响, 其中花寿命作为 因变量, 授粉处理作为固定效应, 物种则作为随机 效应; 而在物种水平上运用单因素方差分析 (ANOVA)检验不同授粉处理对花寿命的影响。

最后, 为了比较不同海拔高度下植物花寿命及 其可塑性的大小变异以及对不同强度的授粉处理 的响应, 在群落水平上, 运用线性混合模型分析不 同海拔下植物花寿命及其可塑性的变异, 其中花寿 命(即潜在花寿命、实际花寿命与最短花寿命)、同 一组植株花寿命的可塑性大小、对套袋处理的响应 以及对授粉处理的响应作为因变量, 海拔作为固定 效应，物种则作为随机效应(Trunschke \& Stöcklin, 2016); 而在物种水平上, 运用 $t$ 检验检验同一物种 在不同海拔下花寿命的变异。

为了统计分析的方便, 将低海拔所有研究物种 组成的群落记为群落1, 将高海拔所有研究物种组 成的群落记为群落 2 , 将两个海拔共有开花物种组 成的低海拔群落和高海拔群落分别记为群落 3 和群 落4。所有的数据分析都在SPSS 19.0中进行。

\section{2 结果}

线性混合模型分析的结果显示, 物种、海拔、 授粉处理以及主要交互作用均显著影响花寿命(表1;
$P<0.05)$ 。

\section{1 不同授粉处理对花寿命的影响}

一般线性模型分析结果显示, 无论在群落1 $\left(F_{2,16}=38.857, P<0.001\right)$ 还是群落2 $\left(F_{2,9}=22.086\right.$, $P<0.001)$ 中，不同的授粉处理对植物花寿命都产 生了显著影响。群落1中，在群落水平上，套袋显著 延长了植物花寿命 $\left(F_{1,184}=85.542, P<0.001\right)$, 而补 充授粉显著缩短了植物花寿命 $\left(F_{1,162}=16.781, P<\right.$ $0.001)$; 而在物种水平上，除蒲公英 $(P=0.175)$ 和野 草莓 $(P=0.759)$ 外, 所有物种的平均潜在花寿命都 显著长于平均实际花寿命; 钝裂银莲花 $(P=$ $0.031)$ 、莓叶委陵菜 $(P=0.027)$ 以及野草莓 $(P=$ $0.017)$ 的实际花寿命显著长于最短花寿命 (表2)。群 落2中, 在群落水平上, 套袋显著延长了植物花寿 命 $\left(F_{1,119}=60.708, P<0.001\right)$, 补充授粉显著缩短了 植物花寿命 $\left(F_{1,106}=43.752, P<0.001\right)$; 在物种水平 上，所有物种的潜在花寿命均显著长于实际花寿命; 除小花草玉梅 $(P=0.571)$ 外, 所有物种的实际花寿 命均显著长于最短花寿命(表2)。

在群落3中, 套袋显著延长了植物花寿命 $\left(F_{1,93}=\right.$ 48.543, $P<0.001)$, 而补充授粉则显著缩短了花寿命 $\left(F_{1,80}=12.588, P=0.001\right)$ 。同样，在群落4中，套袋 显著延长了花寿命 $\left(F_{1,100}=55.648, P<0.001\right)$, 而补

表1 物种、海拔(低海拔与高海拔)、处理(套袋、补充授粉及自然授粉)及它们的交互作用对群落 1 和 2 以及群落 3 和 4 花寿命的 影响。群落1和 2 分别指低海拔和高海拔所有研究物种组成的群落, 群落3和4分别指低海拔和高海拔共有的研究物种组成的群 落。运用一般线性混合效应模型检验这些因素的显著性并用粗体表示差异显著。

Table 1 The effect of species identity, elevation (low vs. high elevation), and pollination treatments (pollinator exclusion, supplemental pollinated, open-pollinated) and their interactions on flower longevity of studied species in community 1 \& (and community $3 \& 4$ ). The community 1 and 2 refer to the community that composed of all the research species at low and high altitudes, respectively, while the community 3 and 4 refer to the community of the mutual research species at low and high altitudes, respectively. Significant differences are examined from general linear mixed effect models at $P<0.05$ and indicated in bold.

\begin{tabular}{|c|c|c|c|c|c|c|}
\hline \multirow[t]{3}{*}{ 因素 Factor } & \multicolumn{6}{|c|}{ 花寿命 Floral longevity } \\
\hline & \multicolumn{3}{|c|}{ 群落1和2 Community 1 \& 2} & \multicolumn{3}{|c|}{ 群落3和4 Community 3 \& 4} \\
\hline & Df & $F$ & $P$ & $D f$ & $F$ & $P$ \\
\hline 截距 Intercept & 1,10 & $17,634.897$ & $<0.001$ & 1,9 & $23,757.518$ & $<0.001$ \\
\hline 物种 Species & 10,434 & 762.000 & $<0.001$ & 4, 269 & $1,121.932$ & $<0.001$ \\
\hline 海拔 Elevation & 1,437 & 50.519 & $<0.001$ & 1,270 & 55.844 & $<0.001$ \\
\hline 处理 Treatment & 2,433 & 200.241 & $<0.001$ & 2, 267 & 182.577 & $<0.001$ \\
\hline 物种×海拔 Species × Elevation & 4, 435 & 1.218 & 0.303 & 4, 269 & 1.350 & 0.252 \\
\hline 物种 $\times$ 处理 Species $\times$ Treatment & 18,433 & 4.776 & $<0.001$ & 7,267 & 5.668 & $<0.001$ \\
\hline 海拔 $\times$ 处理 Elevation $\times$ Treatment & 2,433 & 4.316 & 0.014 & 2,267 & 4.722 & 0.010 \\
\hline $\begin{array}{l}\text { 物种 } \times \text { 海拔 } \times \text { 处理 } \\
\text { Species } \times \text { Elevation } \times \text { Treatment }\end{array}$ & 7,433 & 0.716 & 0.659 & 7, 267 & 0.791 & 0.596 \\
\hline
\end{tabular}


表2 高海拔 $(3,600 \mathrm{~m})$ 和低海拔 $(2,900 \mathrm{~m})$ 的 16 个研究种群在套袋、自然授粉和补充授粉处理下的平均花寿命(天，平均值土标 准差), 同行不同字母表示在同一海拔下不同处理之间花寿命差异显著 $(\boldsymbol{P}<\mathbf{0 . 0 5})$ 。

Table 2 Flower longevity ( $d$, mean \pm SD) of 16 research populations when flowers were either excluded from pollinators, open-pollinated or supplemental hand-pollinated at low $(2,900 \mathrm{~m})$ and high $(3,600 \mathrm{~m})$ elevations. Different letters in the same row at each floral longevity of different treatments indicate significant difference $(P<0.05)$.

\begin{tabular}{|c|c|c|c|c|c|c|}
\hline \multirow[t]{3}{*}{ 物种 Species } & \multicolumn{6}{|c|}{ 花寿命 Floral longevity } \\
\hline & \multicolumn{3}{|c|}{ 低海拔 Low elevation (2,900 m ) } & \multicolumn{3}{|c|}{ 高海拔 High elevation (3,500 m) } \\
\hline & $\begin{array}{l}\text { 套袋处理 } \\
\text { Pollinator } \\
\text { exclusion }\end{array}$ & $\begin{array}{l}\text { 自然授粉 } \\
\text { Open } \\
\text { pollination }\end{array}$ & $\begin{array}{l}\text { 补充授粉 } \\
\text { Supplemental } \\
\text { pollination }\end{array}$ & $\begin{array}{l}\text { 套袋处理 } \\
\text { Pollinator } \\
\text { exclusion }\end{array}$ & $\begin{array}{l}\text { 自然授粉 } \\
\text { Open } \\
\text { pollination }\end{array}$ & $\begin{array}{l}\text { 补充授粉 } \\
\text { Supplemental } \\
\text { pollination }\end{array}$ \\
\hline 蒲公英 Taraxacum mongolicum & $5.2 \pm 0.8^{\mathrm{a}}$ & $4.7 \pm 0.6^{\mathrm{a}}$ & - & $6.5 \pm 1.2^{\mathrm{a}}$ & $5.6 \pm 0.8^{\mathrm{b}}$ & - \\
\hline 甘青老鹳草 Geranium pylzowianum & $4.4 \pm 0.5^{\mathrm{a}}$ & $3.2 \pm 0.4^{\mathrm{b}}$ & $2.8 \pm 0.3^{b}$ & $5.0 \pm 0.5^{\mathrm{a}}$ & $3.8 \pm 0.3^{b}$ & $3.0 \pm 0.3^{\mathrm{c}}$ \\
\hline 针裂银莲花 Anemone obtusiloba & $9.8 \pm 0.9^{\mathrm{a}}$ & $8.6 \pm 1.1^{\mathrm{b}}$ & $7.6 \pm 0.4^{\mathrm{c}}$ & $10.8 \pm 0.8^{\mathrm{a}}$ & $9.4 \pm 0.7^{\mathrm{b}}$ & $7.5 \pm 0.6^{c}$ \\
\hline 莓叶委陵菜 Potentilla fragarioides & $3.5 \pm 0.4^{\mathrm{a}}$ & $2.8 \pm 0.3^{b}$ & $2.4 \pm 0.3^{\mathrm{c}}$ & $3.8 \pm 0.4^{\mathrm{a}}$ & $3.3 \pm 0.3^{b}$ & $2.8 \pm 0.5^{\mathrm{c}}$ \\
\hline 高原毛茛 Ranunculus tanguticus & $9.4 \pm 0.4^{\mathrm{a}}$ & $8.2 \pm 0.8^{\mathrm{b}}$ & $7.7 \pm 1.1^{\mathrm{b}}$ & $10.2 \pm 0.7^{\mathrm{a}}$ & $8.9 \pm 0.8^{\mathrm{b}}$ & $7.8 \pm 0.6^{\mathrm{c}}$ \\
\hline 狼毒 Stellera chamaejasme & $14.3 \pm 1.4^{\mathrm{a}}$ & $11.8 \pm 1.4^{\mathrm{b}}$ & - & - & - & - \\
\hline 鹅线委陵菜 Potentilla anserina & $4.5 \pm 0.6^{\mathrm{a}}$ & $3.2 \pm 0.5^{\mathrm{b}}$ & $3.1 \pm 0.6^{\mathrm{b}}$ & - & - & - \\
\hline 野草莓 Fragaria vesca & $4.4 \pm 0.7^{\mathrm{a}}$ & $4.3 \pm 0.8^{\mathrm{a}}$ & $3.5 \pm 0.5^{\mathrm{b}}$ & - & - & - \\
\hline 华西委陵菜 Potentilla potaninii & $3.3 \pm 0.5^{\mathrm{a}}$ & $2.5 \pm 0.6^{\mathrm{b}}$ & $2.2 \pm 0.3^{b}$ & - & - & - \\
\hline 高山豆 Tibetia himalaica & $4.1 \pm 0.9^{\mathrm{a}}$ & $3.0 \pm 0.5^{\mathrm{b}}$ & $2.8 \pm 0.5^{b}$ & - & - & - \\
\hline $\begin{array}{l}\text { 小花草玉梅 Anemone rivularis var. } \\
\text { flore-minore }\end{array}$ & - & - & - & $5.3 \pm 0.8^{\mathrm{a}}$ & $4.6 \pm 0.6^{\mathrm{b}}$ & $4.4 \pm 1.0^{\mathrm{b}}$ \\
\hline
\end{tabular}

充授粉显著缩短了花寿命 $\left(F_{1,87}=58.196, P<0.001\right)$ 。

\section{2 不同海拔下植物花寿命的变异}

\subsection{1 海拔对植物花寿命的影响}

不同物种的花寿命明显不同，群落1中物种的 平均实际花寿命在 $2.5 \pm 0.6$ 天(华西委陵菜)到 $11.8 \pm$ 1.4 天(狼毒)之间变化, 而群落 2 中则在 $3.3 \pm 0.3$ 天 (莓叶委陵菜)到 $9.4 \pm 0.7$ 天(针裂银莲花)之间变化 (表2)。运用线性混合模型分析发现, 群落2的实际花 寿命 $\left(F_{1,148}=25.315, P<0.001\right)$ 和潜在花寿命 $\left(F_{1,151}\right.$ $=27.418, P<0.001)$ 均显著长于群落 1 , 但最短花寿 命没有显著差异 $\left(F_{1,118}=1.264, P=0.263\right)$ 。

\subsection{2 不同海拔种群间花寿命的变异}

运用 $t$ 检验的结果显示, 除莓叶委陵菜 $(P=$ 0.089)外, 其余共有物种的潜在花寿命均存在显著 差异, 且表现为高海拔的潜在花寿命长于低海拔的 (图1A); 而除莓叶委陵菜 $(P=0.001)$ 外, 其余共有 物种的最短花寿命都没有显著差异(图1B); 除钝裂 银莲花 $(P=0.072)$ 和高原毛茛 $(P=0.081)$ 外, 其余共 有物种高海拔的实际花寿命显著长于低海拔的(图 1C)。在群落水平上，运用线性混合模型分析发现， 低海拔与高海拔共有物种组成的群落中, 群落4的 实际花寿命 $\left(F_{1,96}=29.431, P<0.001\right.$; 图2 $)$ 和潜在 花寿命 $\left(F_{1,97}=30.706, P<0.001\right.$; 图2)均显著长于群
落3的, 但最短花寿命没有显著差异 $\left(F_{1,72}=1.165, P\right.$ $=0.284$; 图2)。

\section{3 不同海拔对花寿命可塑性的影响}

运用线性混合模型分析发现，群落1和群落2中， 花寿命对套袋处理的响应没有显著差异 $\left(F_{1,145}=\right.$ $0.004, P=0.949)$, 说明在这两种海拔高度下，套袋 处理对植物花寿命可塑性大小的影响无显著差异。 而在群落2中，植物花寿命对补充授粉的响应显著 增强 $\left(F_{1,108}=9.055, P=0.003\right)$, 说明对植物进行补 充授粉时，高海拔的植物花寿命缩短的更多。同时， 在群落2中的植物花寿命的最大变化范围显著高于 群落1 $\left(F_{1,124}=7.349, P=0.008\right)$, 说明高海拔植物 的花寿命可塑性更大。与群落1和群落2有类似的结 果：在群落3和群落4中, 植物花寿命对套袋处理的 响应没有显著的差异 $\left(F_{1,95}=0.114, P=0.736\right.$; 图 $3 \mathrm{~A})$; 群落4的物种的花寿命对补充授粉的响应显著 增强 $\left(F_{1,71}=11.703, P=0.001\right.$; 图3B $)$; 在高海拔下 的物种花寿命的可塑性更大 $\left(F_{1,72}=6.614, P=0.012\right.$; 图3C)。

\section{3 讨论}

许多研究已经证明了植物的花寿命与花粉落 置的数量呈负相关, 即在一定范围内花粉落置的数 

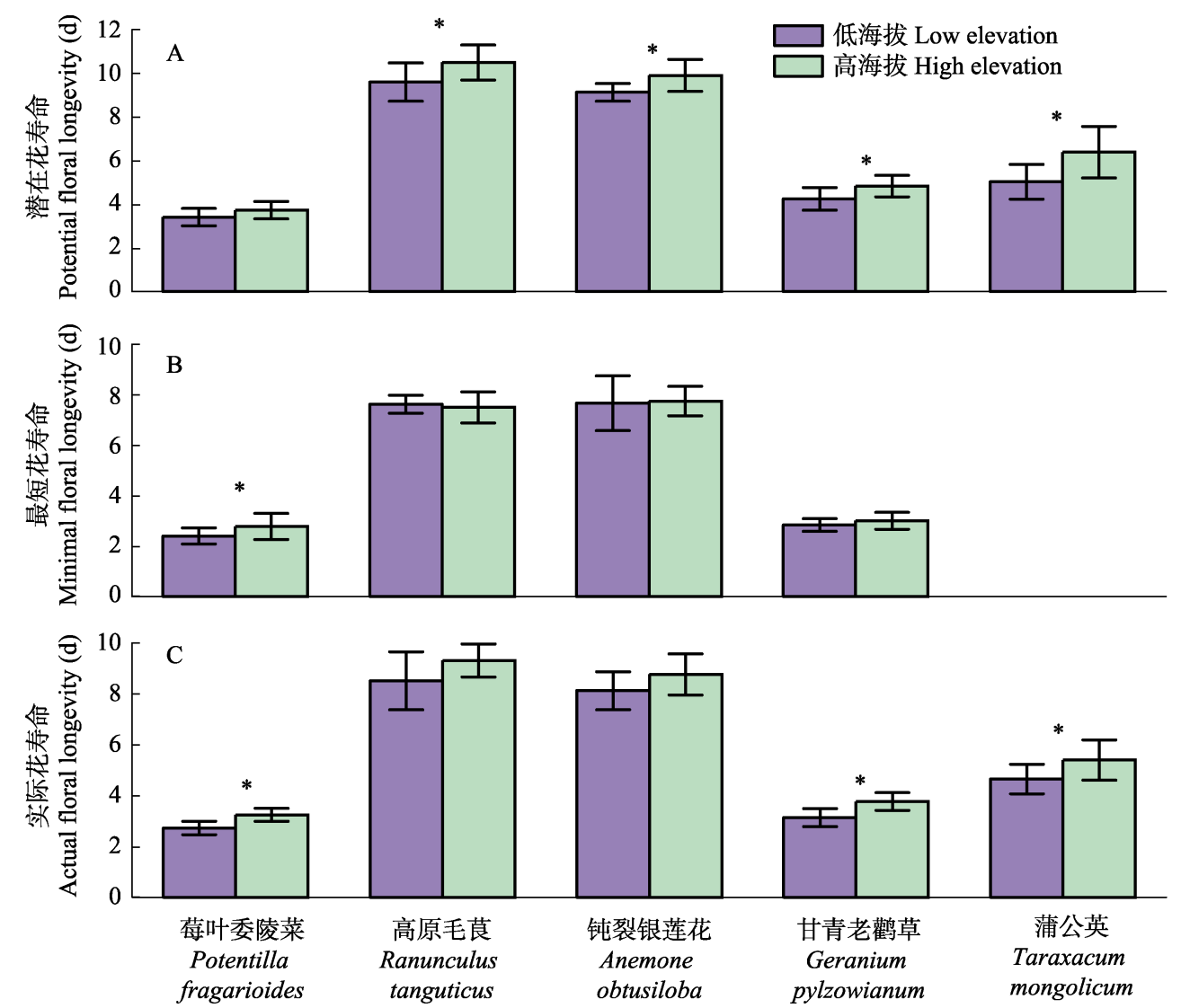

图1 高海拔 $(3,600 \mathrm{~m})$ 和低海拔 $(2,900 \mathrm{~m}) 5$ 种共有物种的平均花寿命(平均值土标准差)的比较。* $\boldsymbol{P}<0.05$ 。

Fig. 1 The mean floral longevity (mean $\pm \mathrm{SD})$ of 5 mutual species at low $(2,900 \mathrm{~m})$ and high $(3,600 \mathrm{~m})$ elevation. $* P<0.05$.

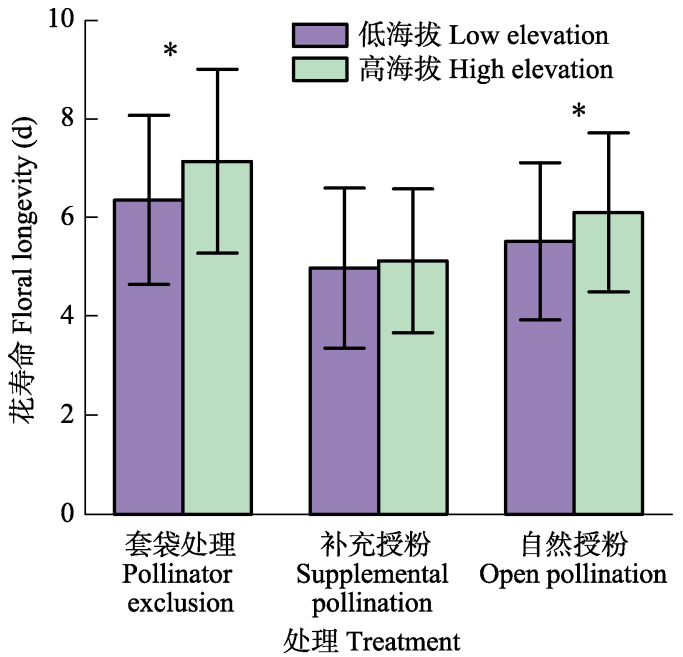

图2 群落3和4的研究物种在套袋、补充授粉以及自然授粉 处理下的平均花寿命(平均值土标准差)的比较。 $* \boldsymbol{P}<0.05$ 。

Fig. 2 The mean flower longevity (mean \pm SD) of community 3 \& 4 when flowers were either pollinator exclusion, supplemental pollination or open pollination. ${ }^{*} P<0.05$.

量越多，该花的寿命就越短(Castro et al, 2008; Arroyo et al, 2013; Spigler, 2017)。同时，一些研究表明,
延长花寿命不仅可以最大化花粉落置的机会, 而且 还可以作为一种竞争机制，即通过增加花展示的大 小来增强其对传粉者的吸引, 进而增加授粉的机会 (Rathcke, 2003; Giblin, 2005)。在本研究中, 实验性 的检验了授粉强度(即花粉落置的数量)对花寿命的 影响，对不同海拔生境的 11 种物种花寿命的野外 观察结果显示, 补充授粉均有缩短植物花寿命的趋 势，套袋处理则均有延长植物花寿命的趋势。而统 计分析的结果表明: 在群落水平, 无论是在高海拔 生境还是低海拔生境，授粉显著地缩短了花寿命， 这与之前相关的报道是一致的(Castro et al, 2008; Arroyo et al, 2013)。然而，在物种水平上，低海拔的 高原毛莨、我线委陵菜、华西委陵菜、高山豆以及 高海拔生境的小花草玉梅的补充授粉处理虽然有 缩短植物花寿命的趋势, 但这种差异不显著。这说 明这些植物种群的实际花寿命已经接近于最短花 寿命了，预示着其花朵的授粉已经接近于最大授粉 强度。无论是在高海拔群落还是低海拔群落套袋处 理都显著地延长了花寿命, 说明套袋处理具有延长 

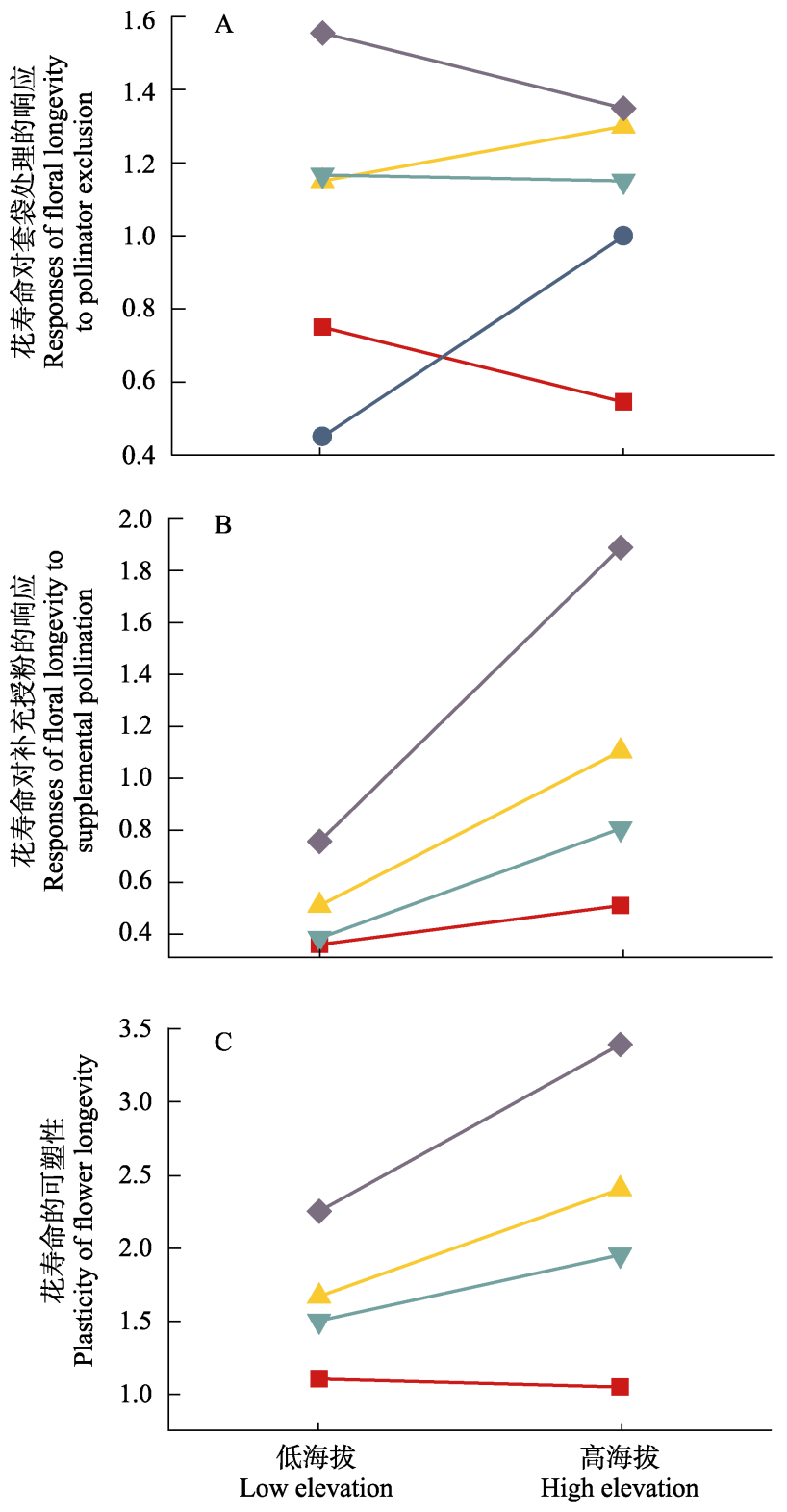

海拔 Elevation

- - 莓叶委陵菜 Potentilla fragarioides

- - 蒲公英 Taraxacum mongolicum 钝裂银莲花 Anemone obtusiloba

- 一 甘青老鹤草 Geranium pylzowianum

——高原毛莨 Ranunculus tanguticus

图3 高海拔和低海拔5种共有物种的花寿命对套袋处理(A) 及补充授粉(B)的响应以及花寿命可塑性(C)的变异。

Fig 3 Differences in responses of floral longevity to pollinator exclusion (A) and supplemental pollination (B) and the plasticity of flower longevity (C)in populations of five mutual plant species from low and high elevation.

花寿命的作用。这与之前的报道一致(Arroyo et al, 2013; Spigler, 2017)。然而, 在物种水平上, 低海拔 的蒲公英和野草莓在套袋处理下, 花寿命的变化不 显著。这说明这两种物种的实际花寿命与潜在花寿
命没有显著差异，预示着在自然状态下昆虫的访花 频率对这两种物种的花寿命的影响不大，这需要进 一步的验证。

传粉环境的差异会影响植物的花寿命(Blionis \& Vokou, 2001; Arroyo et al, 2013), 而海拔作为一 个综合因素, 可以反映传粉环境的差异(Arroyo et al, 1981; Bingham \& Orthner, 1998)。在低海拔生境中, 由于温度较高且传粉者类型、丰富度及活力较高, 植物的雌雄适合度很快就能获得，从而导致植物的 实际花寿命较短; 在高海拔生境中，由于传粉者稀 缺且不稳定，会导致植物的实际花寿命较长 (Primack, 1985; Ashman \& Schoen, 1994)。本研究发 现在群落水平上，高海拔生境植物的实际花寿命和 潜在花寿命均显著高于低海拔生境，但是不同海拔 群落的最短花寿命却没有显著差异, 这可能与物种 最短花寿命的遗传特性有关，即植物的最短雄性功 能期没有显著的差异(Evanhoe \& Galloway, 2002)。 这说明在高海拔下植物花寿命的延长主要是由于 雌性期的延长，这与Gerlinde和Johanna (2010)对 7 种高山植物的研究结果一致: 当花朵被访问的频率 很低时, 植物会通过延长柱头可授性和增加花冠保 鲜时间来延长花寿命。然而, 在物种水平上, 高海 拔和低海拔生境的钝裂银莲花和高原毛茛的实际 花寿命没有显著的差异(表2，图1C)。这是因为这两 种毛莨科植物是自交亲和物种(赵志刚和杜国祯, 2003), 而自交亲和的繁殖对策很好地回避了高山 地区缺乏传粉者的影响，使得植物在极端环境条件 下能不依靠生物媒介而实现自身的繁殖保障(何亚 平等，2005)。因而相对于自交不亲和物种来说，自 交亲和物种的花寿命受传粉环境的影响不大。 Weber和Goodwillie (2013)的研究中有类似的报道。 可见植物的花寿命与植物的交配系统有关，自交亲 和物种的花较早衰老有益于降低资源消耗，而分配 更多的资源进行种子的发育。莓叶委陵菜在高海拔 的最短花寿命显著长于低海拔，这说明其在高海拔 下的最短雄性期发生了延长, 这可能是因为高海拔 生境温度较低，植物发育缓慢的缘故。同时这也有 可能是由于高海拔传粉者低的访花频率导致雄性 适合度很难获得，只能通过延长最短花寿命来保障 雄性适合度(Vesprini \& Pacini, 2005), 但这仍需要 进一步的验证。而莓叶委陵菜的潜在花寿命在海拔 间的差异不显著, 这可能与植物的最长花寿命的遗 
传特性有关(Rathcke, 2003)。这些花寿命的变异都 与群落水平上的研究恰好相反, 说明在物种水平上, 植物的花寿命并不完全符合Ashman和Schoen (1994) 中关于高海拔地区的花寿命延长的理论假设, 而与 植物的交配系统以及植物本身的特性有关。

本研究的结果证明了之前的预测, 即在高海拔 的开花植物群落中, 花寿命对补充授粉的响应更 快。这是因为在高海拔环境中, 传粉者的丰富度及 活力都比较低, 大部分柱头都处于待授粉状态(何 亚平等, 2005), 当对植物进行补充授粉时, 其雌性 适合度实现的速率较快, 从而较早地诱导花朵衰 老。相反, 对低海拔生境中的植物进行补充授粉时, 其雌性适合度实现的速率就相对缓慢。但与本研究 预测不同的是，高海拔和低海拔植物花寿命对套袋 处理的响应没有显著差异。理论上, 相对于高海拔 生境, 低海拔生境中植物未授粉的柱头较少, 所以 植物花寿命对套袋处理的响应更敏感。事实上, 套 袋处理引起的植物花寿命延长主要是延长了花朵 雌性期, 而这种延长是需要消耗资源的。在低海拔 生境, 由于温度相对较高, 使得植物的发育(Arroyo et al, 1981, 2013; Rathcke, 2003)及对水分的消耗(主 要为呼吸作用与蒸腾作用, Teixido \& Valladares, 2015)等都会增加, 而植物为了保障有更多的资源 用于种子的生产，可能会选择通过用新花代替维持 原有花朵的策略(Schoen \& Ashman, 1995; Ashman \& Schoen, 1997), 或通过自交和无融合生殖策略来 保障繁殖, 故而花寿命对隔离传粉者的响应不显 著。但可能的机制需要进一步的研究来检验。总之, 植物花寿命的可塑性主要是由授粉强度引起的, 这 与Trunschke和Stöcklin (2016)的报道一致。

综上, 本研究发现在高海拔生境中, 植物花寿 命的可塑性更大; 而且高海拔生境中, 授粉强度, 或者说主要是传粉者的访花频率影响花寿命的可 塑性。花寿命的可塑性越大代表植物对传粉者稀缺 的不稳定的传粉环境具有更强的适应能力, 植物在 该传粉环境中获得了更大的适合度, 为植物增加了 更多的授粉机会，从而保障了植物的繁殖。

\section{参考文献}

Arroyo MTK, Armesto JJ, Villagran C (1981) Plant phenological patterns in the high Andrean Cordillera of central Chile. Journal of Ecology, 69, 205-223.

Arroyo MTK, Dudley LS, Jespersen G, Pacheco DA, Cavieres
LA (2013) Temperature-driven flower longevity in a highalpine species of Oxalis influences reproductive assurance. New Phytologist, 200, 1260-1268.

Ashman TL, Schoen DJ (1994) How long should flowers live? Nature, 371, 788-791.

Ashman TL, Schoen DJ (1997) The cost of floral longevity in Clarkia tembloriensis: An experimental investigation. Evolutionary Ecology, 11, 289-300.

Aximoff IA, Freitas L (2010) Is pollen removal or seed set favoured by flower longevity in a hummingbird-pollinated Salvia species? Annals of Botany, 106, 413-419.

Bingham RA, Orthner AR (1998) Efficient pollination of alpine plants. Nature, 391, 238-239.

Blionis GJ, Vokou D (2001) Pollination ecology of Campanula species on Mt. Olympos, Greece. Ecography, 24, 287-297.

Castro S, Silveira P, Navarro L (2008) Effect of pollination on floral longevity and costs of delaying fertilization in the out-crossing Polygala vayredae Costa (Polygalaceae). Annals of Botany, 102, 1043-1048.

Clark MJ, Husband BC (2007) Plasticity and timing of flower closure in response to pollination in Chamerion angustifolium (Onagraceae). International Journal of Plant Sciences, 168, 619-625.

Duan YW, Zhang TF, Liu JQ (2007) Interannual fluctuations in floral longevity, pollinator visitation and pollination limitation of an alpine plant (Gentiana straminea Maxim, Gentianaceae) at two altitudes in the Qinghai-Tibetan Plateau. Plant Systematics \& Evolution, 267, 255-265.

Evanhoe L, Galloway LF (2002) Floral longevity in Campanula americana (Campanulaceae): A comparison of morphological and functional gender phases. American Journal of Botany, 89, 587-591.

Gao J, XiongYZ, Huang SQ (2015) Effects of floral sexual investment and dichogamy on floral longevity. Journal of Plant Ecology, 8, 116-121.

Gerlinde S, Johanna W (2010) Flower longevity and duration of pistil receptivity in high mountain plants. Flora, 205, 376-387.

Giblin DE (2005) Variation in floral longevity between populations of Campanula rotundifolia (Campanulaceae) in response to fitness accrual rate manipulation. American Journal of Botany, 92, 1714-1722.

Harder LD, Johnson SD (2005) Adaptive plasticity of floral display size in animal-pollinated plants. Proceedings of the Royal Society of London B: Biological Sciences, 272, 2651-2657.

He YP, Fei SM, Liu JQ, Chen XM, Wang P, Jiang JM (2005) A preliminary review of studies of alpine plant breeding system. Journal of Sichuan Forestry Science and Technology, 26(4), 43-49. (in Chinese with English abstract) [何亚 平，费世民，刘建全，陈秀明，王鹏，蒋俊明 (2005) 高 山植物繁育系统研究进展初探. 四川林业科技, 26(4), 43-49.] 
Hu C, Liu ZJ, Wu GQ, Zhao ZG (2013) Floral characteristic and breeding system of Anemone obtusiloba. Acta Agrestia Sinica, 21, 783-788. (in Chinese with English abstract) [胡 春, 刘左军, 伍国强, 赵志刚 (2013) 针裂银莲花花部综 合特征及其繁育系统. 草地学报, 21, 783-788.]

Itagaki T, Sakai S (2006) Relationship between floral longevity and sex allocation among flowers within inflorescences in Aquilegia buergeriana var. oxysepala (Ranunculaceae). American Journal of Botany, 93, 1320-1327.

Marques I, Draper D (2012) Pollination activity affects selection on floral longevity in the autumnal-flowering plant, Narcissus serotinus L. Botany, 90, 283-291.

Primack RB (1985) Longevity of individual flowers. Annual Review of Ecology and Systematics, 16, 15-37.

Rathcke BJ (2003) Floral longevity and reproductive assurance: Seasonal patterns and an experimental test with Kalmia latifolia (Ericaceae). American Journal of Botany, 90, 1328-1332.

Schoen DJ, Ashman TL (1995) The evolution of floral longevity: Resource allocation to maintenance versus construction of repeated parts in modular organisms. Evolution, 49, 131-139.

Spigler RB (2017) Plasticity of floral longevity and floral display in the self-compatible biennial Sabatia angularis (Gentianaceae): Untangling the role of multiple components of pollination. Annals of Botany, 119, 167-176.

Stratton DA (1989) Longevity of individual flowers in a Costa Rican cloud forest: Ecological correlates and phylogenetic constraints. Biotropica, 21, 308-318.

Sultan SE (2000) Phenotypic plasticity for plant development, function and life history. Trends in Plant Science, 5, 537-542.

Teixido AL, Valladares F (2015) Temperature-limited floral longevity in the large-flowered Mediterranean shrub Cistus ladanifer (Cistaceae). International Journal of Plant Sciences, 176, 131-140.

Trunschke J, Stöcklin J (2016) Plasticity of flower longevity in alpine plants is increased in populations from high elevation compared to low elevation populations. Alpine Botany, 127, 41-51.

Vega Y, Marques I (2015) Both biotic and abiotic factors influence floral longevity in three species of Epidendrum (Orchidaceae). Plant Species Biology, 30, 184-192.

Vesprini JL, Pacini E (2005) Temperature-dependent floral longevity in two Helleborus species. Plant Systematics and Evolution, 252, 63-70.

Weber JJ, Goodwillie C (2013) Variation in floral longevity in the genus Leptosiphon: Mating system consequences. Plant Biology, 15, 220-225.

Zhang J (2013) Study on Reproductive Biology of Taraxacum Species in Northeast China. PhD dissertation, Shenyang Agricultural University, Shenyang. (in Chinese with English abstract) [张建 (2013) 蒲公英属植物繁殖生物学研究. 博士学位论文, 沈阳农业大学, 沈阳.]

Zhang ZQ, Li QJ (2009) Review of evolutionary ecology of floral longevity. Chinese Journal of Plant Ecology, 33, 598-606. (in Chinese with English abstract) [张志强, 李庆 军 (2009) 花寿命的进化生态学意义. 植物生态学报, 33, 598-606.]

Zhao ZG, Du GZ (2003) Characteristics of the mating system and strategies for resource allocation in Ranunculaceae. Journal of Lanzhou University (Natural Sciences), 39(5), 70-74. (in Chinese with English abstract) [赵志刚, 杜国祯 (2003) 毛茛科植物交配系统的特征与花期资源分配对 策. 兰州大学学报(自然科学版), 39(5), 70-74.]

(责任编委：黄双全 责任编辑：黄祥忠) 